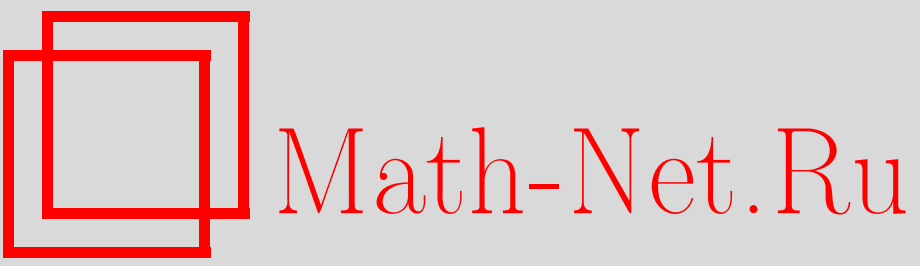

Л. И. Данилов, О спектре двумерного периодического оператора Дирака, ТМФ, 1999, том 118, номер 1, 3-14

DOI: https://doi.org/10.4213/tmf682

Использование Общероссийского математического портала Math-Net.Ru подразумевает, что вы прочитали и согласны с пользовательским соглашением

http://www.mathnet.ru/rus/agreement

Параметры загрузки:

IP : 52.23 .180 .231

26 апреля 2023 г., 14:19:19 


\section{О СПЕКТРЕ ДВУМЕРНОГО ПЕРИОДИЧЕСКОГО ОПЕРАТОРА ДИРАКА}

Доказана абсолютная непрерывность спектра оператора Дирака в $\mathbf{R}^{2}$ с периодическими (с общей решеткой периодов) скалярным $V$ и векторным $A=\left(A_{1}, A_{2}\right)$ потенциалами, для которых $V, A_{j} \in L_{\mathrm{loc}}^{q}\left(\mathbf{R}^{2}\right), q>2$.

Пусть

$$
\widehat{D}=\widehat{D}_{0}+V-\sum_{j=1}^{2} A_{j} \widehat{\alpha}_{j}=\sum_{j=1}^{2} \widehat{\alpha}_{j}\left(-i \frac{\partial}{\partial x_{j}}-A_{j}\right)+\widehat{\beta} m+V
$$

- оператор Дирака в $\mathbf{R}^{2}$, где $m \in \mathbf{R}, \widehat{\alpha}_{j}$ и $\widehat{\beta}$ - эрмитовы $(M \times M)$-матрицы, удовлетворяющие антикоммутационным соотношениям

$$
\widehat{\alpha}_{i} \widehat{\alpha}_{j}+\widehat{\alpha}_{j} \widehat{\alpha}_{i}=2 \delta_{i j} \widehat{I}, \quad \widehat{\alpha}_{i} \widehat{\beta}+\widehat{\beta} \widehat{\alpha}_{i}=\widehat{O}, \quad \widehat{\beta}^{2}=\widehat{I},
$$

$\widehat{O}$ и $\widehat{I}$ - нулевая и единичная матрицы, $\delta_{i j}$ - символ Кронекера. Конкретный вид матриц $\widehat{\alpha}_{j}$ и $\widehat{\beta}$ выбираться не будет (это могут быть матрицы Дирака или матришы Паули). Скалярный (электрический) потенциал $V: \mathbf{R}^{2} \rightarrow \mathbf{R}$ и компоненты векторного (магнитного) потенциала $A_{j}: \mathbf{R}^{2} \rightarrow \mathbf{R}$ - периодические функции с решеткой периодов $\Lambda \subset \mathbf{R}^{2}$. Координаты в $\mathbf{R}^{2}$ задаются относительно ортогонального базиса $\left\{\mathcal{E}_{j}\right\}, j=1,2$. Базис обратной решетки $\Lambda^{*}$ образуют векторы $E_{i}^{*}$, удовлетворяюшие условиям $\left(E_{i}^{*}, E_{j}\right)=\delta_{i j}$, где $\left\{E_{j}\right\}$ - базис решетки $\Lambda$. Положим $K=\mathbf{R}^{2} / \Lambda$,

$$
K^{*}=\left\{k=\eta_{1} E_{1}^{*}+\eta_{2} E_{2}^{*}: 0 \leqslant \eta_{i}<1, i=1,2\right\},
$$

$s(K)$ и $s\left(K^{*}\right)$ - площади двумерного тора $K$ и элементарной ячейки $K^{*}$ решетки $\Lambda^{*}$.

Через $|\cdot|$ и $(\cdot, \cdot)$ обозначаются длина и скалярное произведение векторов в $\mathbf{R}^{2}, \mathcal{M}_{M}-$ линейное пространство (комплексных) $(M \times M)$-матриц с нормой

$$
\|L\|_{\mathcal{M}_{M}}=\max _{\|c\|_{\mathbf{C}^{M}=1}}\|L c\|_{\mathbf{C}^{M}}, \quad L \in \mathcal{M}_{M}
$$

Используются стандартные обозначения для непрерьвных, бесконечно дифференцируемых, интегрируемых в $p$-й степени и (в сушественном) ограниченных функций $f: \mathcal{O} \rightarrow \mathcal{P}$ :

\footnotetext{
* Физико-технический институт УрО РАН, Ижевск, Россия
} 
$C(\mathcal{O} ; \mathcal{P}), C^{\infty}(\mathcal{O} ; \mathcal{P}), L^{p}(\mathcal{O} ; \mathcal{P}), p \geqslant 1$ или $p=\infty$, где $\mathcal{O}=K, \mathbf{R}^{n}, n \geqslant 1$, и $\mathcal{P}=\mathbf{C}^{M}$, $\mathcal{M}_{M}, \mathbf{C}$ (в последнем случае применяется сокрашенная запись $C(\mathcal{O}), C^{\infty}(\mathcal{O})$ и $L^{p}(\mathcal{O})$ ), $L_{\text {lос }}^{p}\left(\mathbf{R}^{2}\right)$ - множество функций $f: \mathbf{R}^{2} \rightarrow \mathbf{C}$, сужение которых на каждое (измеримое) ограниченное множество $\mathcal{O} \subset \mathbf{R}^{2}$ принадлежит пространству $L^{p}(\mathcal{O})$. Скалярные произведения $(\cdot, \cdot)$ и нормы $\|\cdot\|$ в пространствах $\mathbf{C}^{M}, L^{2}\left(\mathbf{R}^{2} ; \mathbf{C}^{M}\right)$ и $L^{2}\left(K ; \mathbf{C}^{M}\right)$ вводятся обычным образом и будут обозначаться одинаково (без указания, как правило, в обозначениях самих пространств $) ; H^{1}\left(\mathbf{R}^{2} ; \mathbf{C}^{M}\right)$ и $H^{1}\left(K ; \mathbf{C}^{M}\right)$ - пространства Соболева квадратично-интегрируемых вместе со своими частными производными первого порядка вектор-функций.

Если $V, A_{j}: \mathbf{R}^{2} \rightarrow \mathbf{R}$ - периодические с решеткой периодов $\Lambda \subset \mathbf{R}^{2}$ функции, для которых $V, A_{j} \in L_{\text {loc }}^{q}\left(\mathbf{R}^{2}\right), j=1,2, q>2$, то оператор $V-A_{1} \widehat{\alpha}_{1}-A_{2} \widehat{\alpha}_{2}$ имеет нулевую грань относительно оператора $\widehat{D}_{0}$, поэтому $\widehat{D}$ - самосопряженньй оператор в гильбертовом пространстве $L^{2}\left(\mathbf{R}^{2} ; \mathbf{C}^{M}\right)$ [1, теорема Х.12] с областью определения $D(\widehat{D})=$ $D\left(\widehat{D}_{0}\right)=H^{1}\left(\mathbf{R}^{2} ; \mathbf{C}^{M}\right)$.

В настоящей работе доказывается следующая теорема.

Теорема 1. Пусть $V, A_{j}: \mathbf{R}^{2} \rightarrow \mathbf{R}$ - периодические с решеткой периодов $\Lambda \subset \mathbf{R}^{2}$ функиии, для которых $V, A_{j} \in L_{\mathrm{loc}}^{q}\left(\mathbf{R}^{2}\right), j=1,2, q>2$. Тогда спектр оператора Дирака $\widehat{D}=\widehat{D}_{0}+V-A_{1} \widehat{\alpha}_{1}-A_{2} \widehat{\alpha}_{2}$ абсолютно непрерывен.

Оператор $\widehat{D}$ унитарно-эквивалентен прямому интегралу

$$
\int_{2 \pi K^{*}}^{\oplus} \widehat{D}(k) \frac{d k}{(2 \pi)^{2} s\left(K^{*}\right)}
$$

где $\widehat{D}(k)=\widehat{D}_{0}(k)+V-A_{1} \widehat{\alpha}_{1}-A_{2} \widehat{\alpha}_{2}$,

$$
\widehat{D}_{0}(k)=\sum_{j=1}^{2} \widehat{\alpha}_{j}\left(-i \frac{\partial}{\partial x_{j}}+k_{j}\right)+\widehat{\beta} m
$$

$k_{j}=\left(k, \mathcal{E}_{j}\right)$, вектор $k \in \mathbf{R}^{2}$ называется квазиимпульсом. Функции $V$ и $A_{j}$ отождествляются с функциями, определенными на торе $K$. В [2] разложение оператора $\widehat{D}$ в прямой интеграл (1) приведено для случая $A_{j} \equiv 0, j=1,2$ (унитарная эквивалентность устанавливается с помошью преобразования Гельфанда [3]), но без всяких изменений оно переносится и на общий случай. Отметим, что область определения $D(\widehat{D}(k))=$ $D\left(\widehat{D}_{0}(k)\right)=H^{1}\left(K ; \mathbf{C}^{M}\right) \subset L^{2}\left(K ; \mathbf{C}^{M}\right)$ операторов $\widehat{D}(k)$ в прямом интеграле $(1)$ не зависит от квазиимпульса $k \in 2 \pi K^{*}$. Оператор $V-A_{1} \widehat{\alpha}_{1}-A_{2} \widehat{\alpha}_{2}$ имеет нулевую грань относительно оператора $\widehat{D}_{0}(k), k \in \mathbf{R}^{2}$, поэтому резольвента самосопряженных операторов $\widehat{D}(k)$ компактна и, следовательно, их спектр дискретен. Пусть $E_{\nu}(k), \nu \in \mathbf{Z}$, - собственные значения операторов $\widehat{D}(k)$ (с учетом их кратности), упорядоченные по возрастанию. Нумерацию собственных значений $E_{\nu}(k)$ при разных $k \in \mathbf{R}^{2}$ можно выбрать так, чтобы функции $\mathbf{R}^{2} \ni k \rightarrow E_{\nu}(k)$ были непрерывными. Спектр оператора $\widehat{D}$ совпадает с $\bigcup_{\nu \in \mathbf{Z}} \Delta_{\nu}$ [4, теорема XIII.85], где $\Delta_{\nu}$ - область значений функции $E_{\nu}(k)$, $k \in 2 \pi K^{*}$. 
Зафиксируем вектор $\gamma \in \Lambda \backslash\{0\}$. Пусть единичный вектор $e \in \mathbf{R}^{2}$ определяется вектором $\gamma: e=|\gamma|^{-1} \gamma$. Для $k \in \mathbf{R}^{2}$ и $\kappa \in \mathbf{R}$ обозначим $\widehat{D}_{0}(k+i \kappa e)=\widehat{D}_{0}(k)+i \kappa\left(e_{1} \widehat{\alpha}_{1}+e_{2} \widehat{\alpha}_{2}\right)$, $\widehat{D}(k+i \kappa e)=\widehat{D}_{0}(k+i \kappa e)+V-A_{1} \widehat{\alpha}_{1}-A_{2} \widehat{\alpha}_{2}$. Операторы $\widehat{D}(k+i \kappa e)$ можно также рассматривать для функций $V, A_{j}: \mathbf{R}^{2} \rightarrow \mathbf{C}, V, A_{j} \in L^{q}(K), q>2 ; D(\widehat{D}(k+i \kappa e))=D\left(\widehat{D}_{0}(k+\right.$ $i \kappa e))=H^{1}\left(K ; \mathbf{C}^{M}\right) ; \widehat{D}_{0}(k+i \kappa e)$ и $\widehat{D}(k+i \kappa e)$ - замкнутые операторы. Обозначим через $a \in \mathbf{R}^{2}$ вектор, для которого

$$
a_{j}=\left(a, \mathcal{E}_{j}\right)=s^{-1}(K) \int_{K} A_{j}(x) d x, \quad j=1,2 .
$$

Для доказательства теоремы 1 воспользуемся методом Томаса [5], с помощью которого доказательство абсолютной непрерывности спектра периодического оператора Дирака сводится к доказательству обратимости и получению оценок для нормы обратных операторов к операторам $\widehat{D}(k+i \kappa e)-\lambda, \lambda \in \mathbf{R}$, при комплексных значениях квазиимпульса $k+i \kappa e \in \mathbf{C}^{2}$. Основным утверждением работы является теорема 2 , из которой теорема 1 вытекает как следствие.

Теорема 2. Пусть функиии $V, A_{j}: K \rightarrow \mathbf{C}, j=1,2$, принадлежат пространству $L^{q}(K), q>2, u \lambda \in \mathbf{C}$. Тогда для любого $\theta \in[0,1)$ найдется (достаточно больиое) число $\kappa>0$ такое, что для всех $k \in \mathbf{R}^{2}:(k-a, e)=\pi|\gamma|^{-1}$ и для всех вектор-функций $\phi \in H^{1}\left(K ; \mathbf{C}^{M}\right)$ справедливо неравенство

$$
\left\|\left(\widehat{D}_{0}(k+i \kappa e)+V-A_{1} \widehat{\alpha}_{1}-A_{2} \widehat{\alpha}_{2}-\lambda\right) \phi\right\| \geqslant \theta \frac{\pi}{|\gamma|} \exp \left\{-c_{1} \sum_{j=1}^{2}\left\|A_{j}-a_{j}\right\|_{L^{q}(K)}\right\}\|\phi\|,
$$

әде константа $c_{1}=c_{1}(q, \Lambda)>0$ зависит только от $q$ и решетки $\Lambda$.

ДОКАЗАТЕЛЬСТВО ТЕОРЕМЫ 1. Из теории возмушений следует, что собственные значения $E_{\nu}(k+\xi e)$ операторов $\widehat{D}(k+\xi e)$ являются аналитическими по $\xi \in \mathbf{R}$ функциями вне их пересечений (без учета кратности). Поэтому из разложения оператора $\widehat{D}$ в прямой интеграл (1) и теорем XIII.85 и XIII.86 из [4] получаем, что для доказательства абсолютной непрерывности спектра оператора $\widehat{D}$ достаточно доказать, что для всех $\nu \in \mathbf{Z}$ и $k \in \mathbf{R}^{2}$ функции $E_{\nu}(k+\xi e), \xi \in \mathbf{R}$, не являются постоянными ни на одном отрезке $\xi \in\left[\xi_{1}, \xi_{2}\right], \xi_{1}<\xi_{2}$. Докажем последнее утверждение. Предположим, что оно неверно. Тогда найдутся число $\lambda \in \mathbf{R}$, вектор $k \in \mathbf{R}^{2}$ и отрезок $\left[\xi_{1}, \xi_{2}\right], \xi_{1}<\xi_{2}$, такие, что оператор $\widehat{D}(k+\xi e)-\lambda$ будет необратимым при всех $\xi \in\left[\xi_{1}, \xi_{2}\right]$. С другой стороны, для любого числа $\xi_{0}+i \kappa_{0} \in \mathbf{C}$ найдутся некоторая окрестность $\mathcal{O}\left(\xi_{0}+i \kappa_{0}\right) \subset \mathbf{C}$ и число $\delta=\delta\left(\xi_{0}, \kappa_{0}\right) \in \mathbf{R}$ такие, что оператор $\widehat{D}_{0}(k+(\xi+i \kappa) e)+\delta$ окажется обратимым для всех $\xi+i \kappa \in \mathcal{O}\left(\xi_{0}+i \kappa_{0}\right)$ (см. лемму 1 в [6]). Так как оператор $V-A_{1} \widehat{\alpha}_{1}-A_{2} \widehat{\alpha}_{2}$ имеет нулевую грань относительно оператора $\widehat{D}_{0}(k)$, то оператор $\left(V-A_{1} \widehat{\alpha}_{1}-A_{2} \widehat{\alpha}_{2}-\right.$ $\lambda-\delta)\left(\widehat{D}_{0}(k+(\xi+i \kappa) e)+\delta\right)^{-1}$ компактен для всех $\xi+i \kappa \in \mathcal{O}\left(\xi_{0}+i \kappa_{0}\right)$. Поэтому из аналитической теоремы Фредгольма [7, теорема VI.14], примененной к операторам $\widehat{I}+\left(V-A_{1} \widehat{\alpha}_{1}-A_{2} \widehat{\alpha}_{2}-\lambda-\delta\right)\left(\widehat{D}_{0}(k+(\xi+i \kappa) e)+\delta\right)^{-1}, \xi+i \kappa \in \mathcal{O}\left(\xi_{0}+i \kappa_{0}\right), \xi_{0}+i \kappa_{0} \in \mathbf{C}$, где $\widehat{I}$ - единичньй оператор, и необратимости оператора $\widehat{D}(k+\xi e)-\lambda$ для всех $\xi \in\left[\xi_{1}, \xi_{2}\right]$ 
следует, что $\lambda$ - собственное значение оператора $\widehat{D}(k+(\xi+i \kappa) e)$ при всех $\xi+i \kappa \in \mathbf{C}$. Последнее противоречит теореме 2 . Теорема 1 доказана.

Использованный при доказательстве теоремы 1 метод Томаса предложен в [5] для доказательства абсолютной непрерывности спектра оператора Шредингера в $\mathbf{R}^{3}$ с периодическим (электрическим) потенциалом $V \in L_{\text {loc }}^{2}\left(\mathbf{R}^{3}\right)$. В [4] результаты работы [5] перенесены на $n$-мерные периодические операторы Шредингера, $n \geqslant 2$. В [8] доказана абсолютная непрерывность спектра двумерного оператора Шредингера с периодическими электрическим потенциалом $V$ и магнитным (векторным) потенциалом $A$, для котоpых $V \in L_{\text {loc }}^{2}\left(\mathbf{R}^{2}\right), A_{j} \in C\left(\mathbf{R}^{2}\right), j=1,2$ (и выполнено условие калибровки $\operatorname{div} A=0$ ). Для оператора Дирака в $\mathbf{R}^{n}$ с периодическим электрическим потенциалом $V$ абсолютная непрерывность спектра доказана в следующих случаях: 1) $n=2, \quad V \in L_{\mathrm{loc}}^{q}\left(\mathbf{R}^{2}\right)$, $q>2[9]$; 2) $n=3, \quad V \in L_{\mathrm{loc}}^{q}\left(\mathbf{R}^{3}\right), q>3[2]$; 3) $n \geqslant 2, V \in C\left(\mathbf{R}^{n}\right)[6,10]$; 4) $n \geqslant 3$ и коэффициенты Фурье $V_{N}$ потенциала $V$ удовлетворяют условию $\sum\left|V_{N}\right|^{p}<+\infty$, где $p=q /(q-1)$ и $q>q(n)>n$; наименьшие значения $q(n)$ (из полученных ранее) приведены в [11]: числа $q(n)$ определяются как корни некоторых алгебраических уравнений четвертой степени, $q(3) \approx 11.645, n^{-2} q(n) \rightarrow 3$ при $n \rightarrow \infty$.

Следующая теорема используется при доказательстве теоремы 2.

ТЕорема 3. Пусть (матричные) функиии $\widehat{W}^{(l)} \in L^{q}\left(K ; \mathcal{M}_{M}\right), \quad l=1,2, \quad q>2$, при (почти) всех $x \in K$ удовлетворяют условиям $\widehat{\alpha}_{j} \widehat{W}^{(1)}(x)=\widehat{W}^{(1)}(x) \widehat{\alpha}_{j} \quad u$ $\widehat{\alpha}_{j} \widehat{W}^{(2)}(x)=-\widehat{W}^{(2)}(x) \widehat{\alpha}_{j}, \quad j=1,2$. Тогда для любого $\theta \in[0,1)$ найдется (достаточно больиое) число $\kappa>0$ такое, что для всех $k \in \mathbf{R}^{2}:(k, e)=\pi|\gamma|^{-1}$ и для всех вектор-функиий $\phi \in H^{1}\left(K ; \mathbf{C}^{M}\right)$ справедливо неравенство

$$
\left\|\left(\widehat{D}_{0}(k+i \kappa e)+\widehat{W}^{(1)}+\widehat{W}^{(2)}\right) \phi\right\| \geqslant \theta \frac{\pi}{|\gamma|}\|\phi\| .
$$

Для функций $\chi \in L^{1}(K ; \mathcal{P})$, где $\mathcal{P}=\mathbf{C}, \mathbf{C}^{M}, \mathcal{M}_{M}$, обозначим

$$
\chi_{N}=s^{-1}(K) \int_{K} \chi(x) e^{-2 \pi i(N, x)} d x, \quad N \in \Lambda^{*} .
$$

Из теоремы вложения Соболева $\left[12\right.$, с. 146] для всех функций $\mathcal{W} \in L^{q}(K), q>2$, и всех вектор-функций $\phi \in H^{1}\left(K ; \mathbf{C}^{M}\right)$ вытекает оценка

$$
\|\mathcal{W} \phi\|_{L^{2}\left(K ; \mathbf{C}^{M}\right)} \leqslant c_{2}\|\mathcal{W}\|_{L^{q}(K)}\|\phi\|_{H^{1}\left(K ; \mathbf{C}^{M}\right)}
$$

где $c_{2}=c_{2}(q, \Lambda)>0$, из которой, в частности, следует, что оператор $V-A_{1} \widehat{\alpha}_{1}-A_{2} \widehat{\alpha}_{2}$ имеет нулевую грань относительно операторов $\widehat{D}_{0}(k), k \in \mathbf{R}^{2}$.

ДоКАЗАТЕЛЬСТво ТЕОРЕмЫ 2. Сделав замену $k-a \rightarrow k$, можно считать, что $(k, e)=\pi|\gamma|^{-1}$ и $\left(A_{j}\right)_{0}=0, j=1,2$. Найдем функции $\Phi^{(n)} \in L^{2}(K), n=1,2$, для которых $\Phi_{0}^{(n)}=0$, так, чтобы выполнялись равенства (в смысле теории распределений)

$$
A_{1}=\frac{\partial \Phi^{(1)}}{\partial x_{1}}+\frac{\partial \Phi^{(2)}}{\partial x_{2}}, \quad A_{2}=\frac{\partial \Phi^{(1)}}{\partial x_{2}}-\frac{\partial \Phi^{(2)}}{\partial x_{1}} .
$$


Для коэффициентов Фурье $\Phi_{N}^{(n)}, N \in \Lambda^{*} \backslash\{0\}$, имеем

$$
2 \pi i \Phi_{N}^{(1)}=\frac{N_{1}}{|N|^{2}}\left(A_{1}\right)_{N}+\frac{N_{2}}{|N|^{2}}\left(A_{2}\right)_{N}, \quad 2 \pi i \Phi_{N}^{(2)}=\frac{N_{2}}{|N|^{2}}\left(A_{1}\right)_{N}-\frac{N_{1}}{|N|^{2}}\left(A_{2}\right)_{N}
$$

где $N_{j}=\left(N, \mathcal{E}_{j}\right), j=1,2$.

ЛЕммА. Функиии

$$
\mathcal{F}^{(j)}(x)=\frac{s^{-1}(K)}{2 \pi i} \sum_{N \in \Lambda^{*} \backslash\{0\}} \frac{N_{j}}{|N|^{2}} e^{2 \pi i(N, x)}, \quad x \in K, \quad j=1,2,
$$

принадлежсат пространствам $L^{p}(K)$ для всех $p \in[1,2)$.

ДокАЗАТЕЛЬСТво ЛЕммы. Будем считать функции $\mathcal{F}^{(j)}$ периодическими в $\mathbf{R}^{2}$ с решеткой периодов $\Lambda$. Пусть

$$
b \in\left(0, \frac{1}{2} \min _{\gamma \in \Lambda \backslash\{0\}}|\gamma|\right) .
$$

Определим функции $G_{b}^{(j)}\left(\xi_{1}, \xi_{2}\right)=\xi_{j}\left(\xi_{1}^{2}+\xi_{2}^{2}\right)^{-1}$, если $\xi_{1}^{2}+\xi_{2}^{2} \leqslant b^{2}$, и $G_{b}^{(j)}\left(\xi_{1}, \xi_{2}\right)=0$, если $\xi_{1}^{2}+\xi_{2}^{2}>b^{2}, \xi_{1}, \xi_{2} \in \mathbf{R}$. Справедливо равенство

$$
\iint_{\mathbf{R}^{2}} G_{b}^{(j)}\left(\xi_{1}, \xi_{2}\right) e^{i\left(\eta_{1} \xi_{1}+\eta_{2} \xi_{2}\right)} d \xi_{1} d \xi_{2}=\frac{2 \pi i \eta_{1}}{\eta_{1}^{2}+\eta_{2}^{2}}\left(1-J_{0}\left(b \sqrt{\eta_{1}^{2}+\eta_{2}^{2}}\right)\right), \quad \eta_{1}, \eta_{2} \in \mathbf{R}
$$

где $J_{0}$ - функция Бесселя первого рода нулевого порядка. Положим

$$
\mathcal{F}^{(j, 1)}\left(x_{1}, x_{2}\right)=\frac{1}{2 \pi} \sum_{n \in \Lambda} G_{b}^{(j)}\left(x_{1}-n_{1}, x_{2}-n_{2}\right), \quad x_{1}, x_{2} \in \mathbf{R} .
$$

Тогда $\mathcal{F}^{(j, 1)}$ - периодическая функция с решеткой периодов $\Lambda \subset \mathbf{R}^{2}$,

$$
\mathcal{F}_{N}^{(j, 1)}=\frac{s^{-1}(K)}{2 \pi i} \frac{N_{j}}{|N|^{2}}\left(1-J_{0}(2 \pi b|N|)\right)
$$

при $N \in \Lambda^{*} \backslash\{0\}$ и $\mathcal{F}_{0}^{(j, 1)}=0$. При этом $\mathcal{F}^{(j, 1)} \in L^{p}(K)$ для всех $p \in[1,2)$. С другой стороны, функция $\mathcal{F}^{(j, 2)}$ с коэффициентами Фурье $\mathcal{F}_{0}^{(j, 2)}=0$ и

$$
\mathcal{F}_{N}^{(j, 2)}=\frac{s^{-1}(K)}{2 \pi i} \frac{N_{j}}{|N|^{2}} J_{0}(2 \pi b|N|)
$$

при $N \in \Lambda^{*} \backslash\{0\}$ принадлежит пространству $L^{2}(K)$. Поэтому $\mathcal{F}^{(j)}=\mathcal{F}^{(j, 1)}+\mathcal{F}^{(j, 2)} \in$ $L^{p}(K)$ для всех $p \in[1,2)$. Лемма доказана.

Из (3) следуют равенства

$$
\Phi^{(1)}=\mathcal{F}^{(1)} * A_{1}+\mathcal{F}^{(2)} * A_{2}, \quad \Phi^{(2)}=\mathcal{F}^{(2)} * A_{1}-\mathcal{F}^{(1)} * A_{2}
$$


$(*-$ свертка на торе $K)$, из которых с помошью неравенства Гельдера и леммы получаем

$$
\left\|\Phi^{(n)}\right\|_{L^{\infty}(K)} \leqslant c_{3} \sum_{j=1}^{2}\left\|A_{j}\right\|_{L^{q}(K)}, \quad n=1,2,
$$

где $c_{3}=c_{3}(q, \Lambda)>0$.

Пусть $A^{(j, l)} \in C^{\infty}(K), j=1,2, l \in \mathbf{N},-$ последовательности функций такие, что $A_{0}^{(j, l)}=0$ и $\left\|A_{j}-A^{(j, l)}\right\|_{L^{q}(K)} \rightarrow 0$ при $l \rightarrow \infty$. Определим функции $\Phi^{(n, l)}, n=1,2$, $l \in \mathbf{N}$, по функциям $A^{(j, l)}, j=1,2$, с помощью формул (4). Имеем $\Phi^{(n, l)} \in C^{\infty}(K)$ и

$$
\left\|\Phi^{(n)}-\Phi^{(n, l)}\right\|_{L^{\infty}(K)} \leqslant c_{3} \sum_{j=1}^{2}\left\|A_{j}-A^{(j, l)}\right\|_{L^{q}(K)}, \quad n=1,2, \quad l \in \mathbf{N} .
$$

Отсюда, в частности, при $l \rightarrow \infty$ следует, что $\Phi^{(n)} \in C(K)$. Справедливо равенство

$$
\begin{aligned}
e^{-i \Phi^{(1, l)}} e^{-i \widehat{\alpha}_{1} \widehat{\alpha}_{2} \Phi^{(2, l)}}(\widehat{D}(k+i \kappa e)-\lambda) e^{i \Phi^{(1, l)}} e^{-i \widehat{\alpha}_{1} \widehat{\alpha}_{2} \Phi^{(2, l)}}= \\
=\widehat{D}_{0}(k+i \kappa e)-\left(A_{1}-A^{(1, l)}\right) \widehat{\alpha}_{1}-\left(A_{2}-A^{(2, l)}\right) \widehat{\alpha}_{2}+ \\
+(V-\lambda+m \widehat{\beta}) e^{-2 i \widehat{\alpha}_{1} \widehat{\alpha}_{2} \Phi^{(2, l)}}-m \widehat{\beta},
\end{aligned}
$$

из которого и из оценок $(2),(5),(6)$ и замкнутости оператора $\widehat{D}(k+i \kappa e)$ вытекает (при $l \rightarrow \infty)$, что

$$
e^{i \Phi^{(1)}} e^{-i \widehat{\alpha}_{1} \widehat{\alpha}_{2} \Phi^{(2)}} \phi=s-\lim _{l \rightarrow \infty} e^{i \Phi^{(1, l)}} e^{-i \widehat{\alpha}_{1} \widehat{\alpha}_{2} \Phi^{(2, l)}} \phi \in D(\widehat{D}(k+i \kappa e))=H^{1}\left(K ; \mathbf{C}^{M}\right)
$$

для любой вектор-функции $\phi \in H^{1}\left(K ; \mathbf{C}^{M}\right)$ и

$$
\begin{aligned}
& e^{-i \Phi^{(1)}} e^{-i \widehat{\alpha}_{1} \widehat{\alpha}_{2} \Phi^{(2)}}(\widehat{D}(k+i \kappa e)-\lambda) e^{i \Phi^{(1)}} e^{-i \widehat{\alpha}_{1} \widehat{\alpha}_{2} \Phi^{(2)}}= \\
& =\widehat{D}_{0}(k+i \kappa e)+\widehat{W}^{(1)}+\widehat{W}^{(2)},
\end{aligned}
$$

где $\widehat{W}^{(1)}=(V-\lambda) \operatorname{ch} 2 \Phi^{(2)}-i m \widehat{\alpha}_{1} \widehat{\alpha}_{2} \widehat{\beta} \operatorname{sh} 2 \Phi^{(2)}, \widehat{W}^{(2)}=m \widehat{\beta}\left(1-\operatorname{ch} 2 \Phi^{(2)}\right)-i \widehat{\alpha}_{1} \widehat{\alpha}_{2} \times$ $(V-\lambda) \operatorname{sh} 2 \Phi^{(2)}, \widehat{\alpha}_{j} \widehat{W}^{(1)}=\widehat{W}^{(1)} \widehat{\alpha}_{j}$ и $\widehat{\alpha}_{j} \widehat{W}^{(2)}=-\widehat{W}^{(2)} \widehat{\alpha}_{j}, j=1,2$. Из возможности сделать замену $A \rightarrow-A$ следует, что для любой вектор-функции $\phi \in H^{1}\left(K ; \mathbf{C}^{M}\right)$ имеем $e^{-i \Phi^{(1)}} e^{i \widehat{\alpha}_{1} \widehat{\alpha}_{2} \Phi^{(2)}} \phi \in H^{1}\left(K ; \mathbf{C}^{M}\right)$. Поэтому теорема 2 следует из равенства $(7)$, теоремы 3 , оценок (5) и

$$
\left\|e^{ \pm i \Phi^{(1)}(x)} e^{-i \widehat{\alpha}_{1} \widehat{\alpha}_{2} \Phi^{(2)}(x)}\right\|_{\mathcal{M}_{M}} \leqslant e^{\left|\Phi^{(1)}(x)\right|+\left|\Phi^{(2)}(x)\right|}, \quad x \in K,
$$

при этом $c_{1}=4 c_{3}$. Теорема 2 доказана. 
ДОКАЗАТЕЛЬСТВО ТЕОРЕМЫ 3. ВоспольЗУемся методом доказательства, предложенным в [9] (в случае $\widehat{W}^{(2)} \equiv \widehat{O}$ и $\widehat{W}^{(1)}=\mathcal{W} \widehat{I}$, где $\widehat{O}$ и $\widehat{I}$ - нулевая и единичная матрицы в $\mathcal{M}_{M}$ и $\left.\mathcal{W} \in L^{q}(K), q>2\right)$. Будет использован также ряд оценок из [13] и [14]. Без ограничения общности считаем, что $m=0$ (можно добавить слагаемое $m \widehat{\beta}$ к (матричной) функции $\left.\widehat{W}^{(2)}\right)$. Выберем число $Q \in \mathbf{N}$ и числа $\delta_{j}, j=1, \ldots, Q$, так, что $0<\delta_{1}<\cdots<\delta_{Q-1}<\delta_{Q}=1 / 2$,

$$
\delta_{1}<\min \left\{\frac{1}{4}, \frac{q-2}{4}\right\}, \quad \delta_{j}-\delta_{j-1}<\frac{1}{4}, \quad \delta_{j}<\frac{q}{2} \delta_{j-1}
$$

при $j=2, \ldots, Q$. Пусть

$$
\frac{1}{2}<\delta<\frac{q}{q+2} .
$$

Через $\widehat{P}^{\mathcal{C}}$, где $\mathcal{C} \subset \Lambda^{*}$, обозначим ортогональный проектор в гильбертовом пространстве $L^{2}\left(K ; \mathbf{C}^{M}\right)$ на подпространство $\left\{\chi \in L^{2}\left(K ; \mathbf{C}^{M}\right): \chi_{N}=0\right.$ при $\left.N \notin \mathcal{C}\right\}$. Будем использовать краткое обозначение $\widehat{P}^{\mathcal{C}} \chi=\chi^{\mathcal{C}}, \chi \in L^{2}\left(K ; \mathbf{C}^{M}\right)$. Для конечных множеств $\mathcal{C} \subset \Lambda^{*}$ через $\mathcal{N}(\mathcal{C})$ обозначается число элементов в $\mathcal{C}$.

Выберем (и зафиксируем) любую функцию $\eta \in C^{\infty}(\mathbf{R})$ такую, что $\eta(\xi)=1$ при $\xi \leqslant 1$, $0 \leqslant \eta(\xi) \leqslant 1$ при $1<\xi \leqslant 2$ и $\eta(\xi)=0$ при $\xi>2$.

Определим функцию

$$
\omega(x)=\frac{1}{(2 \pi)^{2}} \int_{\mathbf{R}^{2}} \eta(|y|) e^{i(y, x)} d y, \quad x \in \mathbf{R}^{2} .
$$

Для $t>0$ положим $\omega_{1 / t}(x)=t^{2} \omega(t x)$.

В дальнейшем функции, заданные на торе $K$, отождествляются с периодическими функциями в $\mathbf{R}^{2}$ с решеткой периодов $\Lambda$. Если $\widehat{W} \in L^{2}\left(K ; \mathcal{M}_{M}\right)$, то

$$
\left(\widehat{W} * \omega_{\frac{1}{t}}\right)(x)=\sum_{N \in \Lambda^{*}} \eta\left(\frac{2 \pi|N|}{t}\right) \widehat{W}_{N} e^{2 \pi i(N, x)}
$$

$\left(*-\right.$ свертка в $\left.\mathbf{R}^{2}\right)$. Найдутся константы $c_{4}=c_{4}(\eta)>0$ и $c_{5}=c_{5}(\Lambda, \eta)>0$ такие, что при $l=1,2$

$$
\begin{gathered}
\left\|\frac{\partial}{\partial x_{j}}\left(\widehat{W}^{(l)} * \omega_{\frac{1}{t}}\right)\right\|_{L^{\infty}\left(K ; \mathcal{M}_{M}\right)} \leqslant c_{4} t\left\|\widehat{W}^{(l)} * \omega_{\frac{1}{t}}\right\|_{L^{\infty}\left(K ; \mathcal{M}_{M}\right)}, \quad t>0, \quad j=1,2, \\
\left\|\widehat{W}^{(l)} * \omega_{\frac{1}{t}}\right\|_{L^{\infty}\left(K ; \mathcal{M}_{M}\right)} \leqslant c_{5} t^{\frac{2}{q}\left\|\widehat{W}^{(l)}\right\|_{L^{q}\left(K ; \mathcal{M}_{M}\right)}, \quad t \geqslant 1}
\end{gathered}
$$

Пусть $E$ - единичный вектор в $\mathbf{R}^{2}$, для которого $(e, E)=0$ (любой из двух векторов). Для $k \in \mathbf{R}^{2}, \kappa \in \mathbf{R}$ и $N \in \Lambda^{*}$ обозначим

$$
\begin{aligned}
& \widehat{D}_{N}(k ; \kappa)=\sum_{j=1}^{2}\left(k_{j}+2 \pi N_{j}+i \kappa e_{j}\right) \widehat{\alpha}_{j}, \\
& G_{N}^{ \pm}(k ; \kappa)=\left((k+2 \pi N, e)^{2}+(|\kappa| \pm|(k+2 \pi N, E)|)^{2}\right)^{\frac{1}{2}},
\end{aligned}
$$


$G_{N}(k ; \kappa)=G_{N}^{-}(k ; \kappa)$, где $N_{j}=\left(N, \mathcal{E}_{j}\right), j=1,2$. Для всех $c \in \mathbf{C}^{M}$ имеем $[6$, лемма $1 ; 14]$

$$
G_{N}(k ; \kappa)\|c\| \leqslant\left\|\widehat{D}_{N}(k ; \kappa) c\right\| \leqslant G_{N}^{+}(k ; \kappa)\|c\|,
$$

при этом

$$
G_{N}(k ; \kappa) \geqslant|(k+2 \pi N, e)| \geqslant \frac{2 \pi}{|\gamma|}\left\langle\frac{1}{2 \pi}(k, \gamma)\right\rangle,
$$

где $\langle\xi\rangle$ - расстояние от $\xi \in \mathbf{R}$ до ближайшего целого числа. Если $(k, e)=\pi|\gamma|^{-1}$, то $G_{N}(k ; \kappa) \geqslant \pi|\gamma|^{-1}$. Для вектор-функции $\phi \in H^{1}\left(K ; \mathbf{C}^{M}\right)$ будем обозначать $\psi=\psi(\phi)=$ $\left(\widehat{D}_{0}(k+i \kappa e)+\widehat{W}^{(1)}+\widehat{W}^{(2)}\right) \phi$. Тогда

$$
\psi_{N}=\widehat{D}_{N}(k ; \kappa) \phi_{N}+\sum_{l=1}^{2} \sum_{n \in \Lambda^{*}} \widehat{W}_{n}^{(l)} \phi_{N-n}, \quad N \in \Lambda^{*} .
$$

Для всех $k \in \mathbf{R}^{2}:(k, e)=\pi|\gamma|^{-1}$, всех $\kappa \geqslant \kappa_{0}=2^{\frac{2}{2 \delta-1}}$ и всех вектор-функций $\phi \in$ $H^{1}\left(K ; \mathbf{C}^{M}\right)$ определим множества

$$
\begin{aligned}
B & =\left\{N \in \Lambda^{*}:\left\|\widehat{D}_{N}(k ; \kappa) \phi_{N}\right\| \geqslant \kappa^{\frac{1}{2}}\left\|\phi_{N}\right\|\right\}, \\
T_{1} & =\left\{N \in \Lambda^{*}: G_{N}(k ; \kappa)<\kappa^{\delta_{1}}\right\} \backslash B, \\
T_{j} & =\left\{N \in \Lambda^{*}: \kappa^{\delta_{j-1}} \leqslant G_{N}(k ; \kappa)<\kappa^{\delta_{j}}\right\} \backslash B, \quad j=2, \ldots, Q .
\end{aligned}
$$

Положим

$$
T=\bigcup_{j=1}^{Q} T_{j}
$$

Пусть $\operatorname{sign} \xi=\xi|\xi|^{-1}, \quad \xi \in \mathbf{R} \backslash\{0\}$. Если $N \in T$, то $(k+2 \pi N, E) \neq 0$. Обозначим $T_{j}^{ \pm}=\left\{N \in T_{j}: \operatorname{sign}(k+2 \pi N, E)= \pm 1\right\}$,

$$
T^{ \pm}=\bigcup_{j=1}^{Q} T_{j}^{ \pm}
$$

Справедливы оценки

$$
\mathcal{N}\left(T_{j}\right) \leqslant \frac{1}{2 \pi} s^{-1}\left(K^{*}\right)(1+o(1)) \kappa^{2 \delta_{j}}, \quad j=1, \ldots, Q .
$$

Здесь и далее величины, обозначаемые через $o(1)$, имеют при $\kappa \rightarrow+\infty$ обычный смысл. Они могут зависеть от решетки $\Lambda$, числа $q$, матричных функций $\widehat{W}^{(1)}$ и $\widehat{W}^{(2)}$, но не будут зависеть от вектора $k \in \mathbf{R}^{2}:(k, e)=\pi|\gamma|^{-1}$ и вектор-функции $\phi \in H^{1}\left(K ; \mathbf{C}^{M}\right)$. Такое же соглашение используется и для вводимых далее констант $c_{\mu}, \mu=6, \ldots, 10$.

Обозначим $B_{1}=\left\{N \in B: G_{N}(k ; \kappa)<\kappa^{1 / 2}\right\}, B_{2}=B \backslash B_{1}$;

$$
\mathcal{N}\left(B_{1}\right) \leqslant \frac{1}{2 \pi} s^{-1}\left(K^{*}\right)(1+o(1)) \kappa
$$


Для $N \in B$ положим $h_{N}=\kappa^{1 / 2}$, если $N \in B_{1}$, и $h_{N}=G_{N}(k ; \kappa)$, если $N \in B_{2}$. Тогда

$$
\left(\sum_{N \in B} h_{N}^{-q}\right)^{\frac{1}{q}} \leqslant c_{6}(1+o(1)) \kappa^{\frac{1}{q}-\frac{1}{2}}
$$

где $c_{6}=c_{6}(q, \Lambda)>0$. Пусть $\tau>2,1<\sigma<2$ и $1 / \tau+1 / q=1 / 2,1 / \sigma+1 / \tau=1$. Для всех $N \in B$ имеем

$$
\kappa^{\frac{1}{2}}\left\|\phi_{N}\right\| \leqslant h_{N}\left\|\phi_{N}\right\| \leqslant\left\|\widehat{D}_{N}(k ; \kappa) \phi_{N}\right\| \leqslant\left\|\psi_{N}\right\|+\sum_{l=1}^{2}\left\|\left(\widehat{W}^{(l)} \phi\right)_{N}\right\| .
$$

Обозначим

$$
B_{3}=\left\{N \in B:\left\|\psi_{N}\right\| \geqslant \sum_{l=1}^{2}\left\|\left(\widehat{W}^{(l)} \phi\right)_{N}\right\|\right\}, \quad B_{4}=B \backslash B_{3} .
$$

Используя неравенства Гельдера и Хаусдорфа-Юнга, получаем

$$
\begin{aligned}
\left\|\phi^{B_{3}}\right\| & \leqslant 2 \kappa^{-\frac{1}{2}}\left\|\psi^{B_{3}}\right\| \\
\left\|\phi^{B_{4}}\right\| & \leqslant s^{\frac{1}{2}}(K)\left(\sum_{N \in B_{4}} h_{N}^{-q}\right)^{\frac{1}{q}}\left(\sum_{N \in B} h_{N}^{\tau}\left\|\phi_{N}\right\|^{\tau}\right)^{\frac{1}{\tau}} \leqslant \\
& \leqslant 2 c_{6} s^{\frac{1}{2}}(K)(1+o(1)) \kappa^{\frac{1}{q}-\frac{1}{2}} \sum_{l=1}^{2}\left(\sum_{N \in \Lambda^{*}}\left\|\left(\widehat{W}^{(l)} \phi\right)_{N}\right\|^{\tau}\right)^{\frac{1}{\tau}} \leqslant \\
& \leqslant 2 c_{6} s^{-\frac{1}{q}}(K)(1+o(1)) \kappa^{\frac{1}{q}-\frac{1}{2}} \sum_{l=1}^{2}\left(\int_{K}\left\|\widehat{W}^{(l)} \phi\right\|_{\mathbf{C}^{M}}^{\sigma} d x\right)^{\frac{1}{\sigma}} \leqslant \\
& \leqslant 2 c_{6} s^{-\frac{1}{q}}(K)\left(\sum_{l=1}^{2}\left\|\widehat{W}^{(l)}\right\|_{L^{q}\left(K ; \mathcal{M}_{M}\right)}\right)(1+o(1)) \kappa^{\frac{1}{q}-\frac{1}{2}}\|\phi\| .
\end{aligned}
$$

Отсюда имеем

$$
\left\|\phi^{B}\right\| \leqslant\left\|\phi^{B_{3}}\right\|+\left\|\phi^{B_{4}}\right\| \leqslant c_{7}(1+o(1)) \kappa^{-\frac{1}{2}}\left(\left\|\psi^{B}\right\|+\kappa^{\frac{1}{q}}\|\phi\|\right)
$$

и, следовательно,

$$
\left\|\phi^{B}\right\| \leqslant c_{7}(1+o(1)) \kappa^{-\frac{1}{2}}\left(\left\|\psi^{B}\right\|+\kappa^{\frac{1}{q}}\left\|\phi^{A}\right\|\right) .
$$

Положим $\widehat{W}^{(l, 1)}=\widehat{W}^{(l)} * \omega_{\kappa}-\delta, \widehat{W}^{(l, 2)}=\widehat{W}^{(l)}-\widehat{W}^{(l, 1)}, \quad \kappa \geqslant \kappa_{0}, \quad l=1,2$. Пусть $N \in T_{j}, j=1, \ldots, Q$. Тогда

$$
\begin{aligned}
& 2 \kappa(1+o(1)) G_{N}(k ; \kappa)\left\|\phi_{N}\right\|^{2}=G_{N}^{+}(k ; \kappa) G_{N}(k ; \kappa)\left\|\phi_{N}\right\|^{2}= \\
&=\left|(k+2 \pi N+i \kappa e)^{2}\right| \cdot\left\|\phi_{N}\right\|^{2}=\left|\left(\phi_{N}, \widehat{D}_{N}^{2}(k ; \kappa) \phi_{N}\right)\right|= \\
&=\left|\left(\widehat{D}_{N}(k ;-\kappa) \phi_{N}, \psi_{N}-\sum_{l=1}^{2}\left(\widehat{W}^{(l)} \phi\right)_{N}\right)\right| \leqslant \\
& \leqslant\left\|\widehat{D}_{N}(k ;-\kappa) \phi_{N}\right\| \cdot\left(\left\|\psi_{N}\right\|+\sum_{l=1}^{2}\left\|\left(\widehat{W}^{(l)} \phi^{B}\right)_{N}\right\|+\sum_{l=1}^{2}\left\|\left(\widehat{W}^{(l, 2)} \phi^{T}\right)_{N}\right\|\right)+ \\
& \quad+\sum_{l=1}^{2}\left|\left(\phi_{N},\left(\left(-i \sum_{j=1}^{2}\left(\frac{\partial}{\partial x_{j}} \widehat{W}^{(l, 1)}\right) \widehat{\alpha}_{j}+\widehat{W}^{(l, 1)} \widehat{D}_{0}(k+i \kappa e)\right) \phi^{T}\right){ }_{N}\right)\right| .
\end{aligned}
$$


Обозначим $b_{1}=\pi|\gamma|^{-1}$ и $b_{j}=\kappa^{\delta_{j-1}}, j=2, \ldots, Q$. Поскольку $\left\|\widehat{D}_{N}(k ;-\kappa) \phi_{N}\right\| \leqslant(2 \kappa+$ $\left.\kappa^{\delta_{j}}\right)\left\|\phi_{N}\right\|$ (при $N \in T_{j}$ ), то из (11) получаем

$$
\begin{aligned}
(1+o(1)) b_{j}\left\|\phi_{N}\right\| \leqslant & \left\|\psi_{N}\right\|+\sum_{l=1}^{2}\left\|\left(\widehat{W}^{(l)} \phi^{B}\right)_{N}\right\|+\sum_{l=1}^{2}\left\|\left(\widehat{W}^{(l, 2)} \phi^{T}\right)_{N}\right\|+ \\
& +\frac{1}{2 \kappa} \sum_{l=1}^{2}\left\|\left(\widehat{W}^{(l, 1)} \widehat{D}_{0}(k+i \kappa e) \phi^{T}\right)_{N}\right\|+ \\
& +\frac{1}{2 \kappa} \sum_{l=1}^{2}\left\|\left(\left(-i \sum_{j=1}^{2}\left(\frac{\partial}{\partial x_{j}} \widehat{W}^{(l, 1)}\right) \widehat{\alpha}_{j}\right) \phi^{T}\right)_{N}\right\| .
\end{aligned}
$$

Следовательно,

$$
\begin{aligned}
(1+o(1)) b_{j}\left\|\phi^{T_{j}}\right\| \leqslant & \left\|\psi^{T_{j}}\right\|+\sum_{l=1}^{2}\left\|\widehat{P}^{T_{j}} \widehat{W}^{(l)} \phi^{B}\right\|+\sum_{l=1}^{2}\left\|\widehat{P}^{T_{j}} \widehat{W}^{(l, 2)} \phi^{T}\right\|+ \\
& +\frac{1}{2 \kappa} \sum_{l=1}^{2}\left\|\widehat{W}^{(l, 1)} \widehat{D}_{0}(k+i \kappa e) \phi^{T}\right\|+ \\
& +\frac{1}{2 \kappa} \sum_{l=1}^{2}\left\|\left(\sum_{j=1}^{2}\left(\frac{\partial}{\partial x_{j}} \widehat{W}^{(l, 1)}\right) \widehat{\alpha}_{j}\right) \phi^{T}\right\|
\end{aligned}
$$

Получим оценки для слагаемых в правой части последнего неравенства. Из (8) и (9) (при $l=1,2$ ) следует

$$
\begin{aligned}
& \frac{1}{2 \kappa}\left\|\widehat{W}^{(l, 1)} \widehat{D}_{0}(k+i \kappa e) \phi^{T}\right\| \leqslant c_{5}\left\|\widehat{W}^{(l)}\right\|_{L^{q}\left(K ; \mathcal{M}_{M}\right)} \kappa^{-\frac{1}{2}+\frac{2 \delta}{q}}\left\|\phi^{T}\right\|, \\
& \frac{1}{2 \kappa}\left\|\left(\sum_{j=1}^{2}\left(\frac{\partial}{\partial x_{j}} \widehat{W}^{(l, 1)}\right) \widehat{\alpha}_{j}\right) \phi^{T}\right\| \leqslant c_{4} c_{5}\left\|\widehat{W}^{(l)}\right\|_{L^{q}\left(K ; \mathcal{M}_{M}\right)} \kappa^{-1+\left(1+\frac{2}{q}\right) \delta}\left\|\phi^{T}\right\| .
\end{aligned}
$$

Используя (10), получаем

$$
\begin{aligned}
& \left\|\widehat{P}^{T_{j}} \widehat{W}^{(l)} \phi^{B}\right\| \leqslant s^{\frac{1}{2}}(K)\left(\sum_{N \in T_{j}} 1\right)^{\frac{1}{q}}\left(\sum_{N \in T_{j}}\left\|\left(\widehat{W}^{(l)} \phi^{B}\right)_{N}\right\|^{\tau}\right)^{\frac{1}{\tau}} \leqslant \\
& \leqslant s^{-\frac{1}{q}}(K) \mathcal{N}^{\frac{1}{q}}\left(T_{j}\right)\left(\int_{K}\left\|\widehat{W}^{(l)} \phi^{B}\right\|_{\mathbf{C}^{M}}^{\sigma} d x\right)^{\frac{1}{\sigma}} \leqslant \\
& \leqslant c_{8}(q, \Lambda)\left\|\widehat{W}^{(l)}\right\|_{L^{q}\left(K ; \mathcal{M}_{M}\right)}(1+o(1)) \kappa^{\frac{2 \delta_{j}}{q}}\left\|\phi^{B}\right\| \leqslant \\
& \leqslant c_{9}(1+o(1)) \kappa^{-\frac{1}{2}+\frac{2 \delta_{j}}{q}}\left(\left\|\psi^{B}\right\|+\kappa^{\frac{1}{q}}\left\|\phi^{T}\right\|\right), \quad l=1,2 .
\end{aligned}
$$


Оценим теперь норму $\left\|\widehat{P}^{T_{j}} \widehat{W}^{(l, 2)} \phi^{T}\right\|, l=1,2$. Имеем

$$
\begin{aligned}
\left\|\widehat{P}^{T_{j}} \widehat{W}^{(l, 2)} \phi^{T}\right\|^{2} & =s(K) \sum_{N \in T_{j}}\left\|\sum_{n: N-n \in T} \widehat{W}_{n}^{(l, 2)} \phi_{N-n}\right\|^{2} \leqslant \\
& \leqslant s(K) \sum_{N \in T_{j}}\left(\sum_{n: N-n \in T}\left\|\widehat{W}_{n}^{(l, 2)}\right\|_{\mathcal{M}_{M}}^{2}\right)\left(\sum_{n: N-n \in T}\left\|\phi_{N-n}\right\|^{2}\right)= \\
& =\left(\sum_{N \in T_{j}} \sum_{n: N-n \in T}\left\|\widehat{W}_{n}^{(l, 2)}\right\|_{\mathcal{M}_{M}}^{2}\right)\left\|\phi^{T}\right\|^{2}= \\
& =\left(\sum_{n \in \Lambda^{*}} S_{j}(n)\left\|\widehat{W}_{n}^{(l, 2)}\right\|_{\mathcal{M}_{M}}^{2}\right)\left\|\phi^{T}\right\|^{2}
\end{aligned}
$$

где $S_{j}(n)$ - количество таких векторов $N \in T_{j}$, для которых $N-n \in T$. Для всех $n \in \Lambda^{*}$ справедлива оценка $S_{j}(n) \leqslant \mathcal{N}\left(T_{j}\right)$. Поскольку $\widehat{W}_{n}^{(l, 2)}=\widehat{O}$ при $2 \pi|n| \leqslant \kappa^{\delta}$, а при любых $N^{\prime}, N^{\prime \prime} \in T^{+}$или $N^{\prime}, N^{\prime \prime} \in T^{-}$выполняются неравенства $2 \pi\left|N^{\prime}-N^{\prime \prime}\right| \leqslant$ $2 \kappa^{1 / 2} \leqslant \kappa^{\delta}$ (т.к. $\kappa \geqslant \kappa_{0}$ ), то $S_{j}(n)\left\|\widehat{W}_{n}^{(l, 2)}\right\|_{\mathcal{M}_{M}}^{2}>0$ только в случае, если существует вектор $N \in \Lambda^{*}$, для которого $N \in T_{j}^{+}, N-n \in T^{-}$или $N \in T_{j}^{-}, N-n \in T^{+}$. Следовательно, в случае $S_{j}(n)\left\|\widehat{W}_{n}^{(l, 2)}\right\|_{\mathcal{M}_{M}}^{2}>0$ имеем

$$
2 \kappa-\kappa^{\delta_{j}}-\kappa^{\frac{1}{2}}<2 \pi|(n, E)|<2 \kappa+\kappa^{\delta_{j}}+\kappa^{\frac{1}{2}} .
$$

Отсюда $|\kappa-\pi|(n, E)|| \leqslant \kappa^{1 / 2}$. Обозначим

$$
\mathcal{F}_{l}(\kappa)=\sum_{n \in \Lambda^{*}:|\kappa-\pi|(n, E)|| \leqslant \kappa^{\frac{1}{2}}}\left\|\widehat{W}_{n}^{(l, 2)}\right\|_{\mathcal{M}_{M}}^{2}, \quad l=1,2 .
$$

Из (16) получаем

$$
\left\|\widehat{P}^{T_{j}} \widehat{W}^{(l, 2)} \phi^{T}\right\| \leqslant \mathcal{N}^{\frac{1}{2}}\left(T_{j}\right) \mathcal{F}_{l}^{\frac{1}{2}}(\kappa)\left\|\phi^{T}\right\|
$$

Так как

$$
\begin{aligned}
\sum_{l=1}^{2} \int_{\kappa_{0}}^{+\infty} \kappa^{-\frac{1}{2}} \mathcal{F}_{l}(\kappa) d \kappa & \leqslant 2 \sum_{l=1}^{2} \sum_{n \in \Lambda^{*}: \pi|(n, E)| \geqslant \kappa_{0}-\kappa_{0}^{\frac{1}{2}}}\left\|\widehat{W}_{n}^{(l, 2)}\right\|_{\mathcal{M}_{M}}^{2} \leqslant \\
& \leqslant 2 M^{2} s^{-1}(K) \sum_{l=1}^{2}\left\|\widehat{W}^{(l)}\right\|_{L^{2}\left(K ; \mathcal{M}_{M}\right)}^{2}<+\infty
\end{aligned}
$$

то сушествуют числа $\kappa_{\nu} \geqslant \kappa_{0}, \nu \in \mathbf{N}$, такие, что $\kappa_{\nu} \rightarrow \infty$ при $\nu \rightarrow+\infty$ и $\mathcal{F}_{l}\left(\kappa_{\nu}\right) \leqslant \kappa_{\nu}^{1 / 2}$ для всех $\nu \in \mathbf{N}$ и $l=1,2$. Будем далее предполагать, что $\kappa=\kappa_{\nu}$ для некоторого $\nu \in \mathbf{N}$. В этом случае

$$
\left\|\widehat{P}^{T_{j}} \widehat{W}^{(l, 2)} \phi^{T}\right\| \leqslant c_{10}(1+o(1)) \kappa^{\delta_{j}-\frac{1}{4}}\left\|\phi^{T}\right\| .
$$


Выберем числа

$$
\rho_{1}=0, \quad \rho_{j} \in\left(\max \left\{\frac{2}{q} \delta_{j}, \delta_{j}-\frac{1}{4}\right\}, \delta_{j-1}\right), \quad j=2, \ldots, Q .
$$

Сложим неравенства (12) при $j=1, \ldots, Q$, предварительно умножив их на $\kappa^{-\rho_{j}}$. Используя оценки (13)-(15) и (17), получаем

$$
(1+o(1)) \sum_{j=1}^{Q} b_{j} \kappa^{-\rho_{j}}\left\|\phi^{T_{j}}\right\| \leqslant \sum_{j=1}^{Q} \kappa^{-\rho_{j}}\left\|\psi^{T_{j}}\right\|+(1+o(1)) \kappa^{-\frac{1}{2}+\frac{2 \delta_{1}}{q}}\left\|\psi^{B}\right\|+o(1)\left\|\phi^{T}\right\| .
$$

Следовательно,

$$
\frac{\pi}{|\gamma|}(1+o(1))\left\|\phi^{T}\right\| \leqslant\|\psi\|
$$

Отсюда и из неравенства (10) вытекает требуемая оценка

$$
\frac{\pi}{|\gamma|}(1+o(1))\|\phi\| \leqslant\|\psi\|
$$

(при $\left.\kappa=\kappa_{\nu} \rightarrow+\infty, \nu \rightarrow+\infty\right)$. Теорема 3 доказана.

\section{Список литературы}

[1] М. Рид, Б. Саймон. Методы современной математической физики. Т. 2. М.: Мир, 1978.

[2] Л. И. Данилов. ТМФ. 1995. Т. 103. № 1. С. 3-22.

[3] И. М. Гельфанд. ДАН СССР. 1950. Т. 73. № 6. С. 1117-1120.

[4] М. Рид, Б. Саймон. Методы современной математической физики. Т. 4. М.: Мир, 1982.

[5] L. E. Thomas. Commun. Math. Phys. 1973. V. 33. P. 335-343.

[6] Л. И. Данилов. ТМФ. 1990. Т. 85. № 1. С. 41-53.

[7] М. Рид, Б. Саймон. Методы современной математической физики. Т. 1. М.: Мир, 1977.

[8] М. Ш. Бирман, Т. А. Суслина. Алгебра и анализ. 1997. Т. 9. № 1. С. 32-48.

[9] Л.И. Данилов. Спектр оператора Дирака с периодическим потенциалом. III. Деп. в ВИНИТИ 10.07.92. № 2252-В92. М.: ВИНИТИ, 1992.

[10] Л.И. Данилов. О спектре оператора Дирака с периодическим потенциалом. Препринт. Свердловск: ФТИ УрО АН СССР, 1987.

[11] Л.И. Данилов. Спектр оператора Дирака с периодическим потенциалом. VI. Деп. в ВИНИТИ 31.12.96. № 3855-В96. М.: ВИНИТИ, 1996.

[12] И. Стейн. Сингулярные интегралы и дифференциальные свойства функций. М.: Мир, 1973.

[13] Л. И. Данилов. Спектр оператора Дирака с периодическим потенциалом. І. Деп. в ВИНИТИ 12.12.91. № 4588-В91. М.: ВИНИТИ, 1991.

[14] Л.И. Данилов. Спектр оператора Дирака с периодическим потенциалом. IV. Деп. в ВИНИТИ 31.01.95. № 264-В95. М.: ВИНИТИ, 1995. 